Running title: ATTENTION MODULATES TIC-RELATED SYMPTOMS

4

9

17 18

\title{
Help or hurt? How attention modulates tics under different conditions
}

Katja Herrmann ${ }^{1}$, Andreas Sprenger ${ }^{2}$, Leoni Baumung ${ }^{1}$, Daniel Alvarez-Fischer ${ }^{1}$, Alexander Münchau ${ }^{1}$, Valerie Brandt ${ }^{3,1}$

\footnotetext{
${ }^{1}$ Institute of Neurogenetics, Center for Brain, Behavior and Metabolism, University of
}

Lübeck, Lübeck, Germany

${ }^{2}$ Department of Neurology and Institute of Psychology II, Center for Brain, Behavior and Metabolism, University of Lübeck, Lübeck, Germany

${ }^{3}$ Department of Psychology, Centre for Innovation in Mental Health, University of Southampton, UK

17 Corresponding author: Valerie Brandt, Highfield campus, building 44, SO17 1BJ Southampton, UK

Phone: 004423805921375

E-mail: V.C.Brandt@soton.ac.uk 


\section{Running title: ATTENTION MODULATES TIC-RELATED SYMPTOMS}

1

4

\section{8}

Abstract

Tourette syndrome is a neuropsychiatric developmental disorder, characterized by tics that are often preceded by an increasingly uncomfortable urge to move. Tic frequency can increase when patients pay attention to their tics, if tics are not suppressed. This study investigates how attentions modulates urge intensity, tic frequency and arousal during free ticcing and tic suppression.

7 Tic frequency (video recording), urge intensity (rating scale) and pupil width (pupillometry as

8 a measure of arousal) were assessed in 23 patients with Tourette syndrome (mean age $33.48 \pm$ 9 12.37; 14 male) during five attention conditions: 1) baseline, 2) watching own tics in a live video-feedback, 3) watching own tics in a previously recorded video, 4) thinking about situations that can trigger tics and 5) thinking about specific, non-tic related stimuli (distraction condition) during: a) free ticcing and b) tic suppression tic states.

Urge intensity and tic frequency increased in the free ticcing condition when patients viewed 4 their own tics live and when they thought about tic-triggering situations. In the tic suppression condition, tic frequency increased when patients watched a video of their tics, thought about their tics or were distracted. Pupil width increased significantly during the live feedback and the video condition compared to baseline in both tic states.

8 Paying attention to own tics can be detrimental when tics are not suppressed. In contrast, paying 9 attention to other stimuli appears detrimental when tics are suppressed, as would be the case 0 during most current behavioural therapy techniques. However, results point to high emotional 1 arousal and patients feeling uncomfortable when seeing themselves tic. The results also suggest 2 that urge intensity is modulated by changes in attention in the same manner as tics and may 3 drive change in tic frequency during free ticcing.

Key words: Tourette syndrome, tics, attention, urge, arousal 


\section{Introduction}

Gilles de la Tourette syndrome (GTS) is a neurodevelopmental disorder, characterized by multiple motor and vocal tics that have been present for more than a year and first occurred before the age of 18 (DSM-5, 2013). Tics are often associated with an uncomfortable premonitory urge (Kwak, Dat Vuong, \& Jankovic, 2003; Leckman, Walker, \& Cohen, 1993; Reese et al., 2014) that increases before a tic is executed and then decreases (Brandt et al., 2016). It can be hypothesized that urges drive tics by creating a vicious circle in which tics are maintained because ticcing provides relief from the urge (Capriotti, Brandt, Turkel, Lee, \& Woods, 2014).

Several successful behavioral interventions have been established to reduce tic severity (Verdellen, Keijsers, Cath, \& Hoogduin, 2004). The habit reversal therapy (HRT) targets premonitory urges and uses them to teach patients to predict their tics (Deckersbach, Rauch, Buhlmann, \& Wilhelm, 2006; Dutta \& Cavanna, 2013; Wilhelm et al., 2003). Tics can then be replaced with alternative counter-movements that are incompatible with the tic and therefore make the execution of the tic impossible. Exposure with response prevention (ERP) aims to train patients to suppress their tics for as long as possible in order to experience that urges are tolerable and may even habituate over time (Capriotti et al., 2014; Verdellen et al., 2008).

Past studies have found that paying attention to tics can influence tic rates when tics are not suppressed by the patient (Brandt, Lynn, Obst, Brass, \& Munchau, 2015; Misirlisoy et al., 2015; O'Connor, St-Pierre-Delorme, Leclerc, Lavoie, \& Blais, 2014). In a questionnaire study, patients with GTS have reported that thoughts about tics and related phenomena can trigger tics (O'Connor et al., 2014). Moreover, it has been experimentally shown that tic rates increased when patients payed attention to their own tics during a motor task (Misirlisoy et al., 2015) or when they viewed themselves tic freely in a mirror (Brandt et al., 2015). In contrast, tic frequency decreased when patients watched a video that showed them not ticcing (Brandt et al., 2015). The findings posit a number of new questions. This study will address some of them: Firstly, according to the ideomotor theory, motor components of actions cannot be clearly distinguished from their effects (e.g. sensory feedback) and activating these effects, for instance by imagining them, will lead to the tendency to execute the action (Carpenter, 1852; James, 1950). We therefore propose that tic frequency will increase when patients view their tics in a previously recorded video, similar to an increase in tic frequency when watching own tics in a mirror. Furthermore, tic frequency will increase as a direct result of thinking about own tics. Moreover, little is known about the processes underlying the change of tic severity following shifts of attention. Given the close relationship between urges and tics (Brandt et al., 2016), we 
1 hypothesize that urges are affected by attention in the same manner as tic frequency and might 2 drive the change in tic frequency.

3 Second, if tics decrease when patients watch a video that does not show tics, it might be possible

4 5 to create attention conditions, in which attention is diverted from tics and thereby decrease tic frequency. The current study tests the hypothesis that tic frequency will decrease when patients direct their attention to non-tic-related thoughts. Attempts to control unwanted or aversive experiences can lead to paradoxical escalation of the same. This mechanism is especially salient in patients with obsessive-compulsive disorders (OCD) and might be applicable to patients with GTS, given that they are considered part of the same spectrum (McElroy, Phillips, \& Keck, 1994). A number of interventions that have been successfully established in patients with OCD are now evaluated for tics. They encompass accepting urges and other symptoms (Franklin, Best, Wilson, Loew, \& Compton, 2011) and mindfulness interventions (Reese et al., 2015), training patients to pay attention to the moment. Acceptance of urges and mindfulness intervention pilot studies both showed successful symptom reduction in patients with tics (Gev et al., 2016; Reese et al., 2015). Another successful attention-based intervention in patients with OCD is the attention training by Wells (Wells, 1990). Patients are trained to pay attention to stimuli unrelated to their symptoms. While the way to achieve this differs from mindfulnessbased approaches, the idea is similar: patients aim to detach from an exaggerated inner involvement with their symptoms. The current study takes instructions from the attention training as a basis to experimentally tests whether tic frequency decreases when patients are instructed to specifically focus on thoughts that are not tic related.

Third, with regard to tic suppression, it has been shown that tic frequency is not modulated by attention when patients are asked to control their tics and perform a motor task at the same time (Misirlisoy et al., 2015). However, it is conceivable that attention may even have beneficial effects on tic control when patients are not performing an additional task. HRT and ERP both rely on training patients to pay attention to and counter-act tics. Hence, we hypothesize that tic frequency will be lower when patients pay attention to their tics during suppression than when they pay attention to other stimuli.

Finally, pupil dilation was assessed to test the role of emotional arousal (Bradley, Miccoli, Escrig, \& Lang, 2008; Partala, Jokiniemi, \& Surakka, 2000) associated with paying attention to own tics and to test whether arousal could be used as a proxy to measure premonitory urges.

\section{Materials and methods}

\section{Participants}


1 The study included 23 adult patients (mean age $33.48 \pm 12.37$; range $=18-66,14$ male) with a

2 GTS diagnosis according to DSM-5 (American Psychiatric Association, 2013). All patients 3 were diagnosed by a GTS specialist (AM) at the University Hospital in Lübeck, Germany. All patients gave their written informed consent to participate in the study. The study complied with the declaration of Helsinki (World Medical Association, 2013) and was reviewed and approved by the local ethics committee. The required sample size was calculated in $\mathrm{G}^{*}$ Power assuming a medium - large effect size based in a previous study (Brandt et al., 2015). Included were patients with a diagnosis of Tourette syndrome $>18$ years. Excluded were patients with a diagnosis of a current psychotic episode or current substance use. No patients were excluded after data collection. We report all manipulations and all measures in the study.

\section{Procedure and set-up}

Patients were asked to sit in a chair in front of a computer screen with a distance of $60 \mathrm{~cm}$ and to focus on the screen during the experiment. A web-camera (Logitech C310) was mounted on top of the screen. An eye-tracking device (EyeLink 1000 Plus, SR Research, Ottawa, ON, Canada) was installed below the screen for the purpose of pupillometry measurements of one eye (sampling rate: $500 \mathrm{~Hz}$, remote desktop mount participant setup, monocular). The study was conducted in an EEG-laboratory to keep light conditions constant for the pupillometry measurements.

A target sticker was placed on the participants' forehead so that the eye-tracker could keep track of the head position, for instance, during head tics or eye blinking tics. This ensures uninterrupted pupil measurements.

The experimental task was presented in two tic state blocks: a) a free ticcing block and b) a tic suppression block. The blocks were presented in the same order for each patient to ensure that there were no carry-over effects from tic suppression unto free ticcing conditions, although these are unlikely (Muller-Vahl, Riemann, \& Bokemeyer, 2014). Each block consisted of 5 attention conditions (Table 1, Figure 1). Patients were informed that they would be instructed by written cues on the screen in front of them to tic freely during the first block of the experiment and to suppress their tics as much as possible for as long as possible during each experimental condition in the second block of the experiment. The written instructions signalled the start of each experimental block. Moreover, patients were informed that they would be asked to focus their attention on different stimuli during the experiment, for which they would receive auditory instructions during each attention condition of the experiment. 
1 Patients were given a $10 \mathrm{~min}$ break after the free block and were then additionally instructed

2 verbally by the experimenter to suppress tics as much as possible during each attention

3 condition in the suppression block. Patients were told that they could tic freely between the

4 attention conditions.

5 Overall, the experiment encompassed 2 tic state blocks x 5 attention conditions, each lasting 3

6 mins, resulting in 30 mins of paying attention to specific stimuli. Patients decided how much

7 time they needed in between the attention conditions in each block. They could press a button

8 to continue when ready and the task took 50-60 mins depending on the length of these breaks.

9 Tics were recorded throughout the experiment, using the web-camera for head and upper body 10 tics and using a Panasonic HDC-TM700 video camera (25 frames per second) for leg and feet tics. After each attention condition, patients were asked to rate the intensity of their current urge to tic on the urge thermometer, a 10-point scale (0-9; Figure 1). A number of clinical questionnaires were filled out following the experiment.

\section{Task}

16 Each block of the experiment (free ticcing, tic suppression) encompassed five attention conditions (Table 1, Figure 1). During each condition, patients received auditory instructions regarding their focus of attention. The five attention conditions were as follows: in (1) the baseline condition, patients received auditory instructions to focus their attention on the screen in front of them, while watching a picture of the empty room with the chair they were sitting on. The same auditory instructions were given in (2) the live feedback condition, while patients watched themselves on the screen via a real-time video feedback from the web-camera and in (3) the video condition, while patients watched a video of themselves ticcing, recorded in condition (2). The picture of the empty room was also shown in (4), the distraction condition, while patients received instructions to focus on various other things (Table 1) and the (5) ticrelated cognition condition, while patients were instructed to focus on uncomfortable aspects of their tics (Table 1). Auditory instructions were played every $25 \mathrm{~s}$, resulting in 6 instructions (each instruction was played twice per 3 min condition).

Prior to the study, the order of the attention conditions was pseudo-randomized with two constraints: the baseline condition was always presented first and the video condition always followed the live feedback condition. The live feedback condition was adapted from a previous study (Brandt et al., 2015) but the baseline condition was slightly altered. Instead of receiving no instructions, patients received auditory instructions to focus on the screen and saw a picture of the empty experimental room. 
1 Instead of using an actual mirror, patients saw themselves tic in real time on the screen in front

2 of them, using a live-feed from a web camera in order to make all conditions as comparable as 3 possible. The live video feedback was recorded and played back to the patients in the video condition. Auditory stimuli for the tic-related cognitions condition were based on items of the “Thinking About Tics Inventory” (THAT) (O'Connor et al., 2014). Items that were rated highly tic-inducing were selected for the auditory instructions of this study. Auditory instructions for the distraction condition were based on the Attention Training by Wells (Wells, 1990) but instead of playing sounds to the participants, they were asked to imagine and focus on particular sounds. The task stimuli and analysis syntax can be found here: https://osf.io/ufx6j/.

The task was programmed in Matlab® (R2016b, using Psychtoolbox (version 3.12, (Brainard, 1997; Kleiner et al., 2007). No part of the study procedures was pre-registered prior to the research being conducted.

\section{Eye-tracking}

Pupil area was recorded monocularly. Valid eye-tracking data could be recorded in 20 patients. Eye-blinks were excluded and the pupil data was z-transformed.

If $>80 \%$ of pupil data collected from a patient in a condition was missing (e.g. of closed eyes), the value collected for this condition was excluded. Overall, data quality was good; the ticrelated cognition condition had the lowest average percentage of valid data (73.7\%). Missing data analysis shows that $1.5 \%$ of data points were missing, $5 \%$ of values were additionally excluded because they were $>80 \%$ invalid. Overall, $6.5 \%$ of data was replaced by the group mean.

\section{Clinical assessment}

GTS symptom severity was assessed using the clinician-rated "Yale Global Tic Severity Scale" (YGTSS; Leckman et al., 1989). The YGTSS is a structured clinical interview assessing tic severity on a scale from 0-50 (YGTSS50) and subjective impairment on a scale from 0-50. Both scores can be combined and can range from 0-100 (YGTSS100). Current ADHD symptoms were assessed with the German "ADHD self-rating scale" (ADHD-SB; Rosler et al., 2004). Premonitory urges were assessed using the validated German version of the "Premonitory Urge for Tics Scale" (PUTS), a 1-4 Likert rating scale (Rössner, Müller-Vahl, \& Neuner, 2010). The PUTS is a 10 item self-rating scale and was developed to assess urge intensity in children with tics (Woods, Piacentini, Himle, \& Chang, 2005). The last item of the scale assesses the ability to suppress tics and has been removed from the PUTS score because it shows only small 
1 correlations with the rest of the scale (Reese et al., 2014; Woods et al., 2005). Symptoms of

2 OCD were measured with the „Obsessive Compulsive Inventory - Revised“ (OCI-R; Foa et al., 3 2002), a self-report scale with good reliability, convergent and divergent validity (Gonner, 4 Leonhart, \& Ecker, 2008; Hajcak, Huppert, Simons, \& Foa, 2004). Tic-related cognitions were assessed with a translated (German, not validated) version of the THAT (O'Connor et al., 2014). Values of the THAT items were recoded into $0=$ never, $1=$ sometimes, $3=$ always so that higher values reflect higher incidence of tics. Furthermore, a meta-cognitions questionnaire (thinking about thoughts) (Cartwright-Hatton \& Wells, 1997) and the Tourette syndrome quality of life scale (GTS-QOL) (Cavanna et al., 2008) were included.

\section{Data analysis}

No part of the study analyses was pre-registered prior to the research being conducted.

\section{Tic frequency, urge intensity and pupil dilation}

Tic frequency was rated and recorded by two independent raters for each condition (Intraclass Correlation Coefficient free $=.95$, suppression $=.89$ ). Both raters were blinded to the conditions during rating as far as possible (tic-related cognitions and distraction conditions could not be blinded due to the audio instructions). Rater B was blind to the hypotheses of the experiment, rater A was not. Analyses were conducted with the mean ratings of rater A and B. A third independent rater $\mathrm{C}$ who was naïve to all aspects of the experiment rated a subset (two thirds) of the videos. These ratings were used to compare interrater reliability with the ratings used for analysis (Intraclass Correlation Coefficients $\mathrm{AC}=.91$ and $\mathrm{BC}=.91$ ).

Complex tics were counted as one tic, each tic in a burst of multiple tics was counted as a single tic. Number of tics were then averaged across both raters and averaged per minute for the following analyses.

Repeated measures 5 [attention (distraction, baseline, video, live feedback, cognition)] x 2 [tic state (free, suppression)] ANOVAs were conducted with tic frequency (controlled for medication intake), urge intensity and pupil dilation as dependent variables. Contrasts were conducted where interactions were not significant and post-hoc t-tests were corrected for multiple comparisons using Bonferroni-Holm.

A curvilinear regression was used in the baseline condition to describe the pattern of pupil width in relation to tics. Clinical questionnaires were used to describe the sample characteristics.

\section{Pupil dilation around tics}


1 Pupil dilatation in relation to tics was evaluated per second for the baseline conditions in the

2 free ticcing and the tic suppression block. Every movement that was rated as a tic by either rater 3 was entered as a tic in the following analyses. Pupil dilation was $z$-standardized per person and 4 extracted from $-5 \mathrm{~s}$ to $-1 \mathrm{~s}$ before the first tic and from $+1 \mathrm{~s}$ to $+5 \mathrm{~s}$ after the last tic of tic bouts, 5 if two or more tics occurred consecutively, as well as for single tics. The extracted pupil dilation tracks were then averaged per person across all tics and then across all patients, resulting in one average tic-related pupil-width track $(-5 \mathrm{~s}$ to $+5 \mathrm{~s})$ for the baseline conditions in the free ticcing block and the tic suppression block respectively. Curvilinear regressions were then performed with pupil width as the dependent variable and time to tic (-5s to $+5 \mathrm{~s})$ as the independent variable. Statistical tests were considered significant if $\mathrm{p} \leq .05 . \eta^{2}$ and $d$ are reported as effect sizes.

\section{Data availability}

The conditions of our ethical approval and consent procedures do not permit us to archive anonymised study data in a public repository. Individuals seeking access to this data should contact the corresponding author (Valerie Brandt) or Alexander Muenchau [alexander.muenchau@neuro.uni-luebeck.de]. Access will be granted to named individuals in accordance with ethical procedures for handling sensitive clinical data, including completion of a data sharing agreement.

\section{Results}

\section{Clinical assessment}

Out of the 23 participants, 7 were taking medication for their tics, 6 had a diagnosis of comorbid ADHD and 3 comorbid OCD. For clinical scores see Table 2, for correlations between questionnaires see Table 3.

\section{Tic frequency, urge intensity and pupil width, modulated by attention}

\section{Tic frequency}

A 5 [attention (distraction, baseline, video, live feedback, tic-related cognition)] x 2 [tic state (free, suppression)] repeated measures ANOVA revealed that tic frequency was higher in the free ticcing than the tic suppression condition $F(1,22)=6.90, p=.015, \eta^{2}=.24$. There was a significant effect for attention $F(4,88)=8.12, p=.001, \eta^{2}=.27$, and a significant interaction between tic suppression and attention $F(4,88)=5.84, p<.001, \eta^{2}=.21$. Including medication as a covariate did not change the results. 
1 Post-hoc tests of the free condition showed that GTS patients had significantly more tics when

2 they saw themselves tic during live feedback compared to the baseline $t(22)=-2.89, p=.009$,

$3 d=-.60$. Patients did not have significantly more tics when they saw a video of themselves

4 ticcing compared to baseline $t(22)=-1.09, p=.287, d=-.22$. Patients had significantly more tics when they thought about their tics compared to the baseline $t(22)=-3.06, p=.006, d=-$ .64 and did not have significantly fewer tics in the distraction condition compared to the baseline, after Bonferroni-Holm correction $t(22)=2.13, p=.045, d=.44$ (Figure 2A).

In the suppression condition, patients did not tic more frequently when they watched a live feedback of their tics compared to the baseline $t(22)=-.68, p=.50, d=-.14$ but did tic significantly more often when they saw a video of their tics $t(21)=-3.14, p=.005, d=-.65$, thought about their tics $t(22)=-4.11, p<.001, d=-.86$ or thought about distraction stimuli $t(22)=-2.24, p=.036, d=-.47$ (Figure 2A). Significances for the distraction conditions did not survive Bonferroni-Holm correction.

\section{Urge rating}

A 5 [attention (distraction, baseline, video, live feedback, cognition)] x 2 [tic state (free, suppression)] repeated measures ANOVA revealed no significant effect for tic state $F(1,22)=$ $1.71, p=.21, \eta^{2}=.07$, a significant effect for attention $F(4,88)=6.57, p=.001, \eta^{2}=.23$, and no significant interaction between attention and tic state $F(4,88)=.44, p=.72, \eta^{2}=.02$. Linear contrasts showed that the urge was higher when patients saw a live feedback of their tics than at baseline $F(1,22)=7.35, p=.013, \eta^{2}=.25$ and when they thought about their own tics compared to the baseline $F(1,22)=12.29, p=.002, \eta^{2}=.36$. The other contrasts did not reach significance (all $F<2.6, p>.1$ ) (Figure 2B).

\section{Pupil dilation}

A 5 [attention (distraction, baseline, video, live feedback, cognition)] x 2 [tic state (free, suppression)] repeated measures ANOVA revealed a significant effect for tic state $F(1,19)=$ $13.58, p=.002, \eta^{2}=.42$, a significant effect for attention $F(4,76)=21.25, p<.001, \eta^{2}=.53$, and no significant interaction between attention and tic state $F(4,76)=.46, p=.76, \eta^{2}=.02$. Linear contrasts showed that the pupil was more dilated when patients saw a live feedback of their tics than at baseline $F(1,19)=26.11, p<.001, \eta^{2}=.58$ and when they saw their own tics in a video compared to the baseline $F(1,19)=51.63, p<.001, \eta^{2}=.73$. The other contrasts did not reach significance (all $F<1, p>$.1) (Figure 2C). 


\section{Association between the measures}

Pearson correlations showed that tic frequency and urge intensity correlated significantly across conditions and participants $(\mathrm{n}=115, r=.36, p>.001)$, the same was not the case for correlations between urge intensity or tic frequency with pupil width (all $R<.12, p>.24)$.

\section{Pupil dilation around tics}

A curvilinear regression in the baseline free ticcing condition showed a significant quadratic association (increase, then decrease) of pupil dilation around tics $\left[F(2,7)=12.81, p=.005, \mathrm{R}^{2}\right.$ $=.79, \mathrm{~b} 1=-.005, \mathrm{~b} 2=-.009]$. In the suppression condition, the association was cubic $[F(3,6)=$ $\left.10.21, p=.009, \mathrm{R}^{2}=.84, \mathrm{~b} 1=.04, \mathrm{~b} 2=-.003, \mathrm{~b} 3=-.001\right]$, indicating that pupil dilation did not decrease back to the baseline level within $5 \mathrm{~s}$ after a tic (Figure 2D).

\section{Discussion}

This study investigated effects of guided attention on tic frequency and urge intensity in patients with GTS. Pupil dilation was assessed as an indicator of emotional arousal and a possible correlate of urge intensity.

When GTS patients were asked to tic freely, urge intensity and tic frequency increased significantly when patients watched themselves tic via live video feedback and when they thought about tics and tic-related processes but not when they watched a previously filmed video of their own tics or when they payed attention to specific non-tic-related stimuli. The results extend and clarify findings from previous studies, showing that tic frequency increases when patients watch themselves tic in a mirror (Brandt et al., 2015), pay attention to their tics while performing a task (Misirlisoy et al., 2015) and that tic-related cognitions are generally associated with increase in tic severity (O'Connor et al., 2014).

The data demonstrate in a controlled experimental setting, that thinking about own tics and urges and how uncomfortable they are, increases urge intensity and tic frequency when tics are not suppressed. Moreover, the study shows that not every type of attention to tics increases tic frequency. Watching own tics in a previously recorded video did not increase urge intensity or tic frequency. This could be due to the specific timing of tics when watching own tics live, or it could be influenced by a sense of control. The urge to execute an action might only occur if a patient tries to control their actions (Brown et al., 2017). When watching a video that has already been recorded, patients may not feel the need to control their actions because the tics they see are not feedback of what they are currently doing. 


\section{Attention and premonitory urge}

2 This is the first study to show that attention can modulate urge intensity. We hypothesized that the urge might drive tic frequency across different attention conditions when tics are not suppressed, an association that might be expected due to the close relationship between urges and tics (Brandt et al., 2016; Leckman et al., 1993; Reese et al., 2014). The data show that urges were indeed modulated in the same manner as tics in the free ticcing condition, underlining their close relationship. This suggests that both phenomena either rely on related neural mechanisms or that urge intensity mediates the relationship between attention and tic frequency, although the data collected in this study does not allow to test this assumption because the temporal aspect of the urge to tic relationship was not assessed. However, we can provide first evidence that tics and urges behave similarly in response to shifts of attention when tics are not suppressed.

While tics are most likely associated with alterations in the basal ganglia (Bronfeld \& Bar-Gad, 2013; Bronfeld, Belelovsky, \& Bar-Gad, 2011; Bronfeld, Yael, Belelovsky, \& Bar-Gad, 2013; Worbe et al., 2010), urges have been related to activation in the SMA (Fried et al., 1991; Ganos, Roessner, \& Munchau, 2013), insula and cingulate cortex (Jackson, Parkinson, Kim, Schuermann, \& Eickhoff, 2011). While the insula might integrate somatosensory and emotional aspects of urges, the urge to move might be driven by abnormally activated motor plans in the SMA (Maia \& Frank, 2011). These activated motor plans then lead to an increased likelihood of action execution, which would be negatively reinforced by ameliorating the urge to move, possibly signalled by the insula. In this case, urges would modulate tic frequency via highly interconnected neural networks.

Urges may particularly arise when tics are anticipated or remembered because the motor plan of a tic is activated as soon as the movement is imagined or anticipated. The ideomotor theory proposed that the tendency to act is afforded by imagining the act (Carpenter, 1852; James, 1950). Actions are not isolated motor events but are a complex interplay of movement, sensory anticipation and feedback (James, 1950). Therefore, activating action effects, such as imagining, anticipating (Elsner \& Hommel, 2001), or remembering motor or sensory information associated with a tic, can trigger the tendency to execute the tic and may evoke the urge to do so. Hence, anticipating to see oneself tic in a mirror or screen and imagining particular tics and feelings associated with tics (tic-related cognitions) may induce the urge to tic. However, the data shows that it is not quite that simple. Seeing own tics in a video should have the same effect because seeing tics should also activate the respective motor plans but patients rated their urge intensity to be no different from the baseline and tic frequency was not 
significantly higher than at baseline. Another hypothesis regarding the urge to execute an action states that an urge arises from the need or want to control said action (Brown et al., 2017). The urge to execute an action will occur when the action is "held in check" (Brown et al., 2017). Patients in this study had control over seeing themselves tic during live feedback. They also had control when recalling uncomfortable tics and urges by acting them out or not. In contrast, seeing their own tics in a previously recorded video may have been uncomfortable for patients but controlling their tics in this condition would not have changed the tics patients watched. Therefore, they may not have experienced an increased urge to tic and, as a consequence, no rise in tic frequency. However, this assumption remains speculative at this point.

Several interesting conclusions can be drawn. Not every form of attention to own tics increases the urge to tic and tic frequency. It seems likely that urges are associated with the attempt to control tics when stored tic-related motor or sensory information are activated, although it is also possible that different mechanisms play a role in different conditions. It would be interesting to compare neural activity preceding tics when tics are viewed in a live video feedback compared to a previously recorded video.

\section{Tic suppression}

Interestingly, urge intensity was influenced by attention in the same manner in the tic suppression condition as in the free ticcing condition, whereas the pattern of results changed with respect to tic frequency. Tic frequency did not differ between the baseline condition and when patients watched their own tics in a live feedback and when they focused on specific nontic related thoughts, while it increased when patients focused on a video of their tics and when they thought about the discomfort associated with tics.

The results suggest that tic suppression is more successful when patients focus on their tics (baseline, live-feedback, tic-related cognitions) than when they have to divide their attention (video, distraction). Interestingly, very little is known about differing techniques or strategies that patients might apply to suppress their tics (e.g. distraction, effortful top-down control). The results of this study are in contrast with a previous study that showed that tic frequency was not modulated by attention when tics were suppressed (Misirlisoy et al., 2015). However, that study used a motor task across all attention conditions and it is therefore difficult to compare with the current study design. Urge intensity was modulated by attention in the same fashion as in the free condition, underlining previous findings that urge intensity and tic frequency become decoupled when tics are suppressed (Brandt et al., 2016). 
1 The results are interesting with respect to behavioural therapy of tics. While paying attention to

2 own tics when tics are not controlled increases tic frequency in some settings, it might help to

3 keep tic frequency low when patients suppress their tics. A recent study showed that therapy success was not predicted by behavioural performance in some cognitive and motor control tasks (Abramovitch et al., 2017), suggesting that inhibitory control might not be a large factor for successful behavioural therapy. Learning to specifically direct attention towards tic-related processes could be a factor related to the effectiveness of behavioural therapy. An interesting question would be whether this is always beneficial because patients learn how to direct their attention towards controlling tics or whether it can also have detrimental effects when tics are not controlled. However, the experimental data collected in this study cannot be directly generalized to therapeutic mechanisms and should therefore be treated with caution in this respect.

Strikingly, overall urge intensity was not reported to be significantly higher in the suppression condition than in the free ticcing condition. Several explanations are possible. There is mixed literature on whether or not urge intensity increases when GTS patients suppress tics (Capriotti et al., 2014; Himle, Woods, Conelea, Bauer, \& Rice, 2007; Muller-Vahl et al., 2014; Specht et al., 2013). It is possible that patients did not experience an increase in urge intensity during tic suppression in addition to the effects attention had on urge intensity. Another explanation concerns the urge thermometer itself. Due to the colour-scheme, patients may have been hesitant to rate their urges as very high, or in the "red zone". Our data would support this notion as none of the patients used the full range of the urge thermometer.

\section{Pupil dilation}

In the baseline condition, pupils dilated before a tic was executed and contracted afterwards. This pattern is very similar to the behaviour of urges around tics (Brandt et al., 2016). However, mean pupil dilation across conditions did not correspond to mean urge intensity as rated by the patients. Therefore, pupil dilation cannot be assumed to be purely a correlate of urge intensity. In general, pupil dilation is associated with emotional arousal, regardless of negative or positive content (Bradley et al., 2008; Partala et al., 2000). Our results indicate that pupil dilation might be a correlate of anxiety or discomfort associated with tics and ticcing, rather than urge intensity.

In line with tic frequency changes, pupils were significantly less dilated in the suppression condition (not more, as would be expected if it correlated with urges), a finding that makes it more likely that pupil dilation also or exclusively corresponds to emotional processes associated 
with tics. This may include but not be restricted to the urge to tic. Furthermore, pupils were significantly more dilated when patients viewed themselves tic compared to the baseline but not in the tic-related cognition condition or the distraction condition. Regarding this result, pupil dilation did not correspond with either urge intensity or tic frequency. Hence, pupil dilation might be influenced by emotional processes not only directly related to executing tics. It could be hypothesized that watching own tics is particularly uncomfortable and cannot be "edited" as memory or imagination. Therefore, the live feedback and video conditions may have been particularly associated with emotional arousal in patients.

In conclusion, pupil dilation cannot be viewed as a correlate of urge intensity across different conditions. Nevertheless, it could be used as a proxy for urge intensity within the same condition, where emotional arousal is kept relatively constant.

\section{Distraction}

Contrary to our hypothesis, tic frequency, urge intensity and pupil dilation did not decrease significantly in the distraction condition compared to the baseline. However, it should be noted that the effect for a decrease in tic frequency was in the medium range and should not be entirely disregarded. The difficulty with using a baseline condition in patients with GTS is that it cannot be exactly defined what patients do or pay attention to during baseline measurements. By always running the baseline condition first, we made sure that patients were not thinking about previously presented attention instructions (at least in the free condition) and were behaving as closely to a "real life" situation as possible during an experiment that was recorded on video. Furthermore, they did receive instruction to pay attention to the screen in front of them. However, it is always difficult to set a "tic baseline" because tics can be influenced by a number of factors.

\section{Limitations}

There are several limitations to the study. Pupil dilation and tic frequency were recorded in an ongoing fashion while the urge to tic was judged in a general manner at the end of each condition. Although this rating should reflect the general impact of the attention condition on the urge to tic, it cannot reflect urge fluctuations during the condition. On the other hand, a tic intensity rating after each tic would have interrupted the experiment frequently. Therefore, the association between urge intensity and pupil dilation should be investigated in an independent task where urges are assessed continuously. 
1 Furthermore, the urge thermometer, especially in combination with using colours, may not be

2 the most sensitive instrument to measure changes in urge intensity, especially in participants

3 that refrain from using extreme ends of scales. Some patients may also have started low on the

4 scale in the baseline condition and were then unable to give comparatively lower ratings after the distraction condition. Future experiments might ask patients to start their urge rating in the baseline condition at 5 per default and then only look at differences between the baseline and the other condition.

According to prior power calculations, the sample size should be sufficient to show a positive effect of cognitive distraction if it was there. However, we noted a number of limitations regarding the distraction condition used in this experiment by asking patients for feedback after the experiment. The main limitation was that the auditory instructions given were not specific enough. For instance, one patient reported having imagined to go into cold water, which the patient felt made them tic more. Therefore, the instructions should be more specific to imagine pleasant situations and could even be individually tailored to situations where patients with GTS feel they tic less frequently or experience an attenuated urge to tic. Another possibility would be to include a motor component in the distraction condition, for instance a fidget toy or a finger exercise. However, it would then be impossible to distinguish processes related to attention and processes related to controlled motor activation and their effect on tics. Motor activation has been shown to be associated with lower tic frequency (Doja et al., 2018; Misirlisoy et al., 2015; Nixon, Glazebrook, Hollis, \& Jackson, 2014). Therefore, a motor intervention would require an active baseline. Regarding this study, it could be argued that some conditions were more active than others, requiring patients to imagine scenes. It is unclear how cognitive activation interacts with tics. However, the conditions were held constant with regard to motor activation and required patients to only switch their focus of attention.

Secondly, we did not control whether patients followed instructions in the distraction condition. While patients reported afterwards that they did, it is possible that their thoughts drifted towards other things during the condition. The attention training used by Wells requires intense concentration and effort and has to be exercised daily for several weeks (Wells, 1990). We would therefore view the results regarding the distraction condition as data to build upon. Although we cannot conclude that distraction decreased tic frequency or urge intensity as compared to the baseline, the effect size is promising enough to try and refine the distraction condition for the purpose of testing whether attention distraction can be used as a therapeutic method to decrease symptom severity in patients with GTS. 
1 Lastly, while we were able to clarify a number of questions with regard to the conditions under

2 which attention modulates tic frequency and urge intensity, we do not know whether the

3 processes underlying these changes are the same in every condition and what they are. The

4 exact underlying (neural) processes need to be further investigated in the future.

5

6 Acknowledgement

7 This work was supported by grants from the Deutsche Forschungsgemeinschaft

8 (DFG): MU 1692/4-1 and FOR 2698 to A.M. We like to thank H.-G. Fehn, University of

9 Applied Sciences Münster/Germany, for supplying 64-bit video capture software

10 "vid_capture" for Matlab®.

11

12 Funding

13 This research did not receive specific funding

14

15 Competing interests

16 None.

17

18

19

20

21

22 
Table 1. Attention instructions

\begin{tabular}{ll}
\hline Attention & English \\
condition &
\end{tabular}

(1) Baseline 11 Please focus your attention Konzentrieren Sie sich bitte auf

(2) live on the screen in front of you den Bildschirm vor Ihnen

feedback 2 Please focus your attention Richten Sie Ihre Konzentration

$\boldsymbol{\&}$ on the video you are bitte auf das Video das Sie sehen

(3) video watching

condition

3 Please look at the screen in Schauen Sie bitte auf den front of you and think about Bildschirm vor Ihnen und denken nothing in particular Sie über nichts Besonderes nach

(4) Distraction 1 Imagine you are taking a Stellen Sie sich vor Sie gehen am walk on the beach and focus Strand spazieren und on the sound of the waves konzentrieren Sie sich auf das Geräusch der Wellen

2 Imagine you are taking a Stellen Sie sich vor Sie gehen im walk in the woods and pay Wald spazieren und achten auf attention to the birds singing den Gesang der Vögel

3 During the next minute, Achten Sie innerhalb der focus your attention on the nächsten Minute auf die sounds surrounding you Geräusche die sie um sich herum wahrnehmen

(5) Tic-related 1 For the duration of the next Denken Sie in der nächsten cognitions minute, please think about your tics and focus on those Minute bitte über Ihre Tics nach that you find most auf die Tics, die sie am meisten bothersome stören

2 Now please think about Denken Sie nun bitte über situations in which you find Situationen nach, in denen Sie 
your tics especially Ihre Tics besonders schlimm oder distressing or exhausting anstrengend finden

3 Please focus on the feeling Konzentrieren Sie sich nun bitte that you have to tic in order darauf, dass Sie ticcen müssen to feel a sensation of relief um ein Gefühl der Erleichterung zu empfinden

1

2 The table shows the different attention instructions (3 different instructions per attention

3 condition, played twice in the order above per attention condition) given to the patients during

4 the five different attention conditions of the experiment (left-most column). Attention

5 instructions were the same in the baseline, live-feedback and video condition (patients were

6 asked to focus on the screen). Instruction changed in the distraction condition (patients were

7 asked to think about specific non-tic related stimuli, based on Wells' attention training) and

8 the tic-related condition (patients were asked to focus on specific tic-related thoughts, based 9 on THAT items). 
Table 2. Questionnaire scores

\begin{tabular}{lllllclll}
\hline Score & YGTSS & YGTSS & $\begin{array}{l}\text { PUTS } \\
\text { ADHD- }\end{array}$ & OCI-R & MCQ 65 & $\begin{array}{l}\text { GTS- } \\
\text { QOL }\end{array}$ & THAT \\
& $\mathbf{5 0}$ & $\mathbf{1 0 0}$ & $\mathbf{9}$ & SB & & & QOL & \\
\hline $\mathbf{M} \pm$ & $19.83 \pm$ & $38.39 \pm$ & $21.13 \pm$ & $15.80 \pm$ & $13.35 \pm$ & $112.54 \pm$ & $22.48 \pm$ & 20.59 \\
SD & 8.10 & 17.38 & 4.99 & 9.94 & 10.71 & 25 & 18.54 & \pm 9.87 \\
Range & $8-45$ & $8-85$ & $14-31$ & $0-40$ & $0-36$ & $65-260$ & & $1-37$ \\
Possible & & & & & & & & \\
range & $0-50$ & $0-100$ & $9-36$ & $0-60$ & $0-72$ & $67-155$ & $1-65$ & $0-66$
\end{tabular}

Above

cut-off

$6 \quad 3$

1

2 The table shows mean clinical scores, standard deviations, the range of clinical scores in this

3 study, as well as the possible range of the questionnaire. YGTSS = Yale Global Tic Severity

4 Scale; The PUTS = Premonitory Urge for Tics Scale; ADHD-SB = German ADHD self-

5 rating scale; OCI-R = Obsessive Compulsive Inventory- Revised; THAT $=$ Thinking About

6 Tics questionnaire (recoded), MCQ = Meta-cognitions questionnaire, Tourette syndrome -

7 quality of life scale (GTS-QOL). OCI-R cut-off $=21$, ADHD-SB cut-off according to DSM-5

8 criteria (Rosler et al., 2004).

9 
Running title: ATTENTION MODULATES TIC-RELATED SYMPTOMS

Table 3. Questionnaire correlations

\begin{tabular}{lccccccc}
\hline Score & YGTSS50 & $\begin{array}{c}\text { YGTSS } \\
\text { impairment }\end{array}$ & PUTS9 & \multicolumn{1}{c}{$\begin{array}{l}\text { ADHD- } \\
\text { SB }\end{array}$} & & $\begin{array}{l}\text { OCI-R } \\
\text { QOL }\end{array}$ & THAT \\
\hline MCQ 65 & $.47^{*}$ & .39 & .37 & $.58^{* *}$ & $.73^{* * *}$ & $.67^{* *}$ & .31 \\
THAT & .07 & $.48^{*}$ & $.45^{*}$ & $.65^{* *}$ & $.57^{* *}$ & $.60^{* *}$ & \\
\hline
\end{tabular}

1

2 The table shows correlations (Spearman's rho) between the metacognitions and the thinking

3 about tics questionnaires and the clinical scales. YGTSS = Yale Global Tic Severity Scale;

4 The PUTS = Premonitory Urge for Tics Scale; ADHD-SB = German ADHD self-rating scale;

5 OCI-R = Obsessive Compulsive Inventory- Revised; THAT $=$ Thinking About Tics

6 questionnaire (recoded), MCQ = Meta-cognitions questionnaire, Tourette syndrome - quality

7 of life scale (GTS-QOL). Significant correlations marked in grey did not survive correction

8 for multiple tests.

$9 * \mathrm{p}<.05$

$10 * * \mathrm{p}<.01$

$11 * * * \mathrm{p}<.001$ 
Running title: ATTENTION MODULATES TIC-RELATED SYMPTOMS

\section{References}

Abramovitch, A., Hallion, L. S., Reese, H. E., Woods, D. W., Peterson, A., Walkup, J. T., . . Wilhelm, S. (2017). Neurocognitive predictors of treatment response to randomized treatment in adults with tic disorders. Progress in Neuro-Psychopharmacology and Biological Psychiatry, 74, 9-14. doi:10.1016/j.pnpbp.2016.11.002

American Psychiatric Association. (2013). Diagnostic and statistical manual of mental disorders: DSM-V (5 ed.). Washington, DC.

Bradley, M. M., Miccoli, L., Escrig, M. A., \& Lang, P. J. (2008). The pupil as a measure of emotional arousal and autonomic activation. Psychophysiology, 45(4), 602-607. doi:10.1111/j.1469-8986.2008.00654.x

Brainard, D. H. (1997). The Psychophysics Toolbox. Spatial Vision, 10(4), 433-436.

Brandt, V. C., Beck, C., Sajin, V., Baaske, M. K., Baumer, T., Beste, C., . . Munchau, A. (2016). Temporal relationship between premonitory urges and tics in Gilles de la Tourette syndrome. Cortex, 77, 24-37. doi:10.1016/j.cortex.2016.01.008

Brandt, V. C., Lynn, M. T., Obst, M., Brass, M., \& Munchau, A. (2015). Visual feedback of own tics increases tic frequency in patients with Tourette's syndrome. Cognitive Neuroscience, 6(1), 1-7. doi:10.1080/17588928.2014.954990

Bronfeld, M., \& Bar-Gad, I. (2013). Tic disorders: what happens in the basal ganglia? Neuroscientist, 19(1), 101-108. doi:10.1177/1073858412444466

1073858412444466 [pii]

Bronfeld, M., Belelovsky, K., \& Bar-Gad, I. (2011). Spatial and temporal properties of ticrelated neuronal activity in the cortico-basal ganglia loop. Journal of Neuroscience, 31(24), 8713-8721. doi:10.1523/JNEUROSCI.0195-11.2011

Bronfeld, M., Yael, D., Belelovsky, K., \& Bar-Gad, I. (2013). Motor tics evoked by striatal disinhibition in the rat. Frontiers in Systems Neuroscience, 7, 50. doi:10.3389/fnsys.2013.00050

Brown, B. J., Kim, S., Saunders, H., Bachmann, C., Thompson, J., Ropar, D., . . . Jackson, G. M. (2017). A Neural Basis for Contagious Yawning. Current Biology. doi:10.1016/j.cub.2017.07.062

Capriotti, M. R., Brandt, B. C., Turkel, J. E., Lee, H. J., \& Woods, D. W. (2014). Negative Reinforcement and Premonitory Urges in Youth With Tourette Syndrome: An 
Running title: ATTENTION MODULATES TIC-RELATED SYMPTOMS

Experimental Evaluation. Behavior Modification, 38(2), 276-296. doi:10.1177/0145445514531015

Carpenter, W. B. (1852). On the influence of suggestion in modifying and directing muscular movement, independently of volition. Proceedings of the Royal Institution, 147-154.

Cartwright-Hatton, S., \& Wells, A. (1997). Beliefs about worry and intrusions: the MetaCognitions Questionnaire and its correlates. Journal of Anxiety Disorders, 11(3), 279296.

Cavanna, A. E., Schrag, A., Morley, D., Orth, M., Robertson, M. M., Joyce, E., . . Selai, C. (2008). The Gilles de la Tourette syndrome-quality of life scale (GTS-QOL): development and validation. Neurology, 71(18), 1410-1416. doi:10.1212/01.wnl.0000327890.02893.61

Deckersbach, T., Rauch, S., Buhlmann, U., \& Wilhelm, S. (2006). Habit reversal versus supportive psychotherapy in Tourette's disorder: a randomized controlled trial and predictors of treatment response. Behaviour Research and Therapy, 44(8), 10791090. doi:10.1016/j.brat.2005.08.007

Doja, A., Bookwala, A., Pohl, D., Rossi-Ricci, A., Barrowman, N., Chan, J., \& Longmuir, P. E. (2018). Relationship Between Physical Activity, Tic Severity and Quality of Life in Children with Tourette Syndrome. Journal of the Canadian Academy of Child and Adolescent Psychiatry. Journal de l'Académie Canadienne de Psychiatrie de L'enfant et de l'Adolescent, 27(4), 222-227.

DSM-5. (2013). Diagnostic and statistical manual of mental disorders (5 ed.). Arlington, VA: American Psychiatric Publishing.

Dutta, N., \& Cavanna, A. E. (2013). The effectiveness of habit reversal therapy in the treatment of Tourette syndrome and other chronic tic disorders: a systematic review. Functional Neurology, 28(1), 7-12. doi:5806 [pii]

Elsner, B., \& Hommel, B. (2001). Effect anticipation and action control. Journal of Experimental Psychology: Human Perception and Performance, 27(1), 229-240.

Foa, E. B., Huppert, J. D., Leiberg, S., Langner, R., Kichic, R., Hajcak, G., \& Salkovskis, P. M. (2002). The Obsessive-Compulsive Inventory: development and validation of a short version. Psychological Assessment, 14(4), 485-496. 
1 Franklin, M. E., Best, S. H., Wilson, M. A., Loew, B., \& Compton, S. N. (2011). Habit Reversal Training and Acceptance and Commitment Therapy for Tourette Syndrome: A Pilot Project. Journal of Developmental and Physical Disabilities, 23(1), 49-60.

Fried, I., Katz, A., McCarthy, G., Sass, K. J., Williamson, P., Spencer, S. S., \& Spencer, D. D. (1991). Functional organization of human supplementary motor cortex studied by electrical stimulation. Journal of Neuroscience, 11(11), 3656-3666.

Ganos, C., Roessner, V., \& Munchau, A. (2013). The functional anatomy of Gilles de la Tourette syndrome. Neuroscience and Biobehavioral Reviews, 37(6), 1050-1062. doi:10.1016/j.neubiorev.2012.11.004

Gev, E., Pilowsky-Peleg, T., GFennig, S., Benaroya-Milshtein, N., Woods, D. W., Piacentini, J., . .. Steinberg, T. (2016). Acceptanceofpremonitoryurgesandtics. Journal of ObsessiveCompulsive and Related Disorders, 10, 78-83.

Gonner, S., Leonhart, R., \& Ecker, W. (2008). The Obsessive-Compulsive Inventory-Revised $(\mathrm{OCl}-\mathrm{R})$ : validation of the German version in a sample of patients with $\mathrm{OCD}$, anxiety disorders, and depressive disorders. Journal of Anxiety Disorders, 22(4), 734-749. doi:10.1016/j.janxdis.2007.07.007

Hajcak, G., Huppert, J. D., Simons, R. F., \& Foa, E. B. (2004). Psychometric properties of the $\mathrm{OCI}-\mathrm{R}$ in a college sample. Behaviour Research and Therapy, 42(1), 115-123.

Himle, M. B., Woods, D. W., Conelea, C. A., Bauer, C. C., \& Rice, K. A. (2007). Investigating the effects of tic suppression on premonitory urge ratings in children and adolescents with Tourette's syndrome. Behaviour Research and Therapy, 45(12), 2964-2976. doi:10.1016/j.brat.2007.08.007

Jackson, S. R., Parkinson, A., Kim, S. Y., Schuermann, M., \& Eickhoff, S. B. (2011). On the functional anatomy of the urge-for-action. Cognitive Neuroscience, 2(3-4), 227-243. doi:10.1080/17588928.2011.604717

James, W. (1950). The priciples of psycholgy (Vol. 2). New York, NY: Dover.

Kleiner, M., Brainard, D., Pelli, D., Ingling, A., Murray, R., \& Broussard, C. (2007). What's new in Psychtoolbox-3. Perception, 36(14), 1-16.

Kwak, C., Dat Vuong, K., \& Jankovic, J. (2003). Premonitory sensory phenomenon in Tourette's syndrome. Movement Disorders, 18(12), 1530-1533. doi:10.1002/mds.10618 
Leckman, J. F., Riddle, M. A., Hardin, M. T., Ort, S. I., Swartz, K. L., Stevenson, J., \& Cohen, D. J. (1989). The Yale Global Tic Severity Scale: initial testing of a clinician-rated scale of tic severity. Journal of the American Academy of Child and Adolescent Psychiatry, 28(4), 566-573. doi:S0890-8567(09)65477-0 [pii]

10.1097/00004583-198907000-00015

Leckman, J. F., Walker, D. E., \& Cohen, D. J. (1993). Premonitory urges in Tourette's syndrome. American Journal of Psychiatry, 150(1), 98-102. doi:doi.org/10.1176/ajp.150.1.98

Maia, T. V., \& Frank, M. J. (2011). From reinforcement learning models to psychiatric and neurological disorders. Nature Neuroscience, 14(2), 154-162. doi:10.1038/nn.2723 $\mathrm{nn} .2723$ [pii]

McElroy, S. L., Phillips, K. A., \& Keck, P. E., Jr. (1994). Obsessive compulsive spectrum disorder. Journal of Clinical Psychiatry, 55 Suppl, 33-51; discussion 52-33.

Misirlisoy, E., Brandt, V., Ganos, C., Tubing, J., Munchau, A., \& Haggard, P. (2015). The Relation Between Attention and Tic Generation in Tourette Syndrome. Neuropsychology, 29(4), 658-665. doi:10.1037/neu0000161

Muller-Vahl, K. R., Riemann, L., \& Bokemeyer, S. (2014). Tourette patients' misbelief of a tic rebound is due to overall difficulties in reliable tic rating. Journal of Psychosomatic Research, 76(6), 472-476. doi:10.1016/j.jpsychores.2014.03.003

Nixon, E., Glazebrook, C., Hollis, C., \& Jackson, G. M. (2014). Reduced Tic Symptomatology in Tourette Syndrome After an Acute Bout of Exercise: An Observational Study. Behavior Modification, 38(2), 235-263. doi:10.1177/0145445514532127

O'Connor, K., St-Pierre-Delorme, M. E., Leclerc, J., Lavoie, M., \& Blais, M. T. (2014). Metacognitions in tourette syndrome, tic disorders, and body-focused repetitive disorder. Canadian Journal of Psychiatry. Revue Canadienne de Psychiatrie, 59(8), 417-425. doi:10.1177/070674371405900804

Partala, T., Jokiniemi, M., \& Surakka, V. (2000). Pupillary responses to emotionally provocative stimuli. Paper presented at the Eye Tracking Research \& Application (ETRA), Palm Beach Gardens, Florida, USA.

Reese, H. E., Scahill, L., Peterson, A. L., Crowe, K., Woods, D. W., Piacentini, J., . . W Wilhelm, S. (2014). The premonitory urge to tic: measurement, characteristics, and correlates in 
Running title: ATTENTION MODULATES TIC-RELATED SYMPTOMS

older adolescents and adults. Behavior Therapy, 45(2), 177-186. doi:10.1016/j.beth.2013.09.002

Reese, H. E., Vallejo, Z., Rasmussen, J., Crowe, K., Rosenfield, E., \& Wilhelm, S. (2015). Mindfulness-based stressreductionforTourettesyndromeandchronictic disorder:apilotstudy. Journal of Psychosomatic Research, 78(3), 293-298.

Rosler, M., Retz, W., Retz-Junginger, P., Thome, J., Supprian, T., Nissen, T., . . Trott, G. E. (2004). [Tools for the diagnosis of attention-deficit/hyperactivity disorder in adults. Self-rating behaviour questionnaire and diagnostic checklist]. Nervenarzt, 75(9), 888895. doi:10.1007/s00115-003-1622-2

Rössner, V., Müller-Vahl, K., \& Neuner, I. (2010). PUTS - premonitory urge tics scale: Fragebogen für Kinder. In K. Müller-Vahl (Ed.), Tourette-Syndrom und andere TicErkrankungen im Kindes- und Erwachsenenalter. Berlin: MWV Medizinische Wissenschaftliche Verlagsgesellschaft.

Specht, M. W., Woods, D. W., Nicotra, C. M., Kelly, L. M., Ricketts, E. J., Conelea, C. A., . . Walkup, J. T. (2013). Effects of tic suppression: ability to suppress, rebound, negative reinforcement, and habituation to the premonitory urge. Behaviour Research and Therapy, 51(1), 24-30. doi:10.1016/j.brat.2012.09.009

Verdellen, C. W., Hoogduin, C. A., Kato, B. S., Keijsers, G. P., Cath, D. C., \& Hoijtink, H. B. (2008). Habituation of premonitory sensations during exposure and response prevention treatment in Tourette's syndrome. Behavior Modification, 32(2), 215-227. doi:10.1177/0145445507309020

Verdellen, C. W., Keijsers, G. P., Cath, D. C., \& Hoogduin, C. A. (2004). Exposure with response prevention versus habit reversal in Tourettes's syndrome: a controlled study. Behaviour Research and Therapy, 42(5), 501-511. doi:10.1016/S00057967(03)00154-2

Wells, A. (1990). Panic disorder in association with relaxation induced anxiety: An attentional training approach to treatment. Behavior Therapy, 21, 273-280.

Wilhelm, S., Deckersbach, T., Coffey, B. J., Bohne, A., Peterson, A. L., \& Baer, L. (2003). Habit reversal versus supportive psychotherapy for Tourette's disorder: a randomized controlled trial. American Journal of Psychiatry, 160(6), 1175-1177.

Woods, D. W., Piacentini, J., Himle, M. B., \& Chang, S. (2005). Premonitory Urge for Tics Scale (PUTS): initial psychometric results and examination of the premonitory urge 
Running title: ATTENTION MODULATES TIC-RELATED SYMPTOMS

phenomenon in youths with Tic disorders. Journal of Developmental and Behavioral

2 Pediatrics, 26(6), 397-403.

Worbe, Y., Gerardin, E., Hartmann, A., Valabregue, R., Chupin, M., Tremblay, L., . . Lehericy,

4

S. (2010). Distinct structural changes underpin clinical phenotypes in patients with

5

Gilles de la Tourette syndrome. Brain, 133(Pt 12), 3649-3660. doi:awq293 [pii]

6 10.1093/brain/awq293

7 World Medical Association. (2013). WMA Declaration of Helsinki - Ethical Principles for

Medical Research Involving Human Subjects. JAMA, 310(20), 2191 - 2194.

9 doi:10.1001/jama.2013.281053

10

11 
2

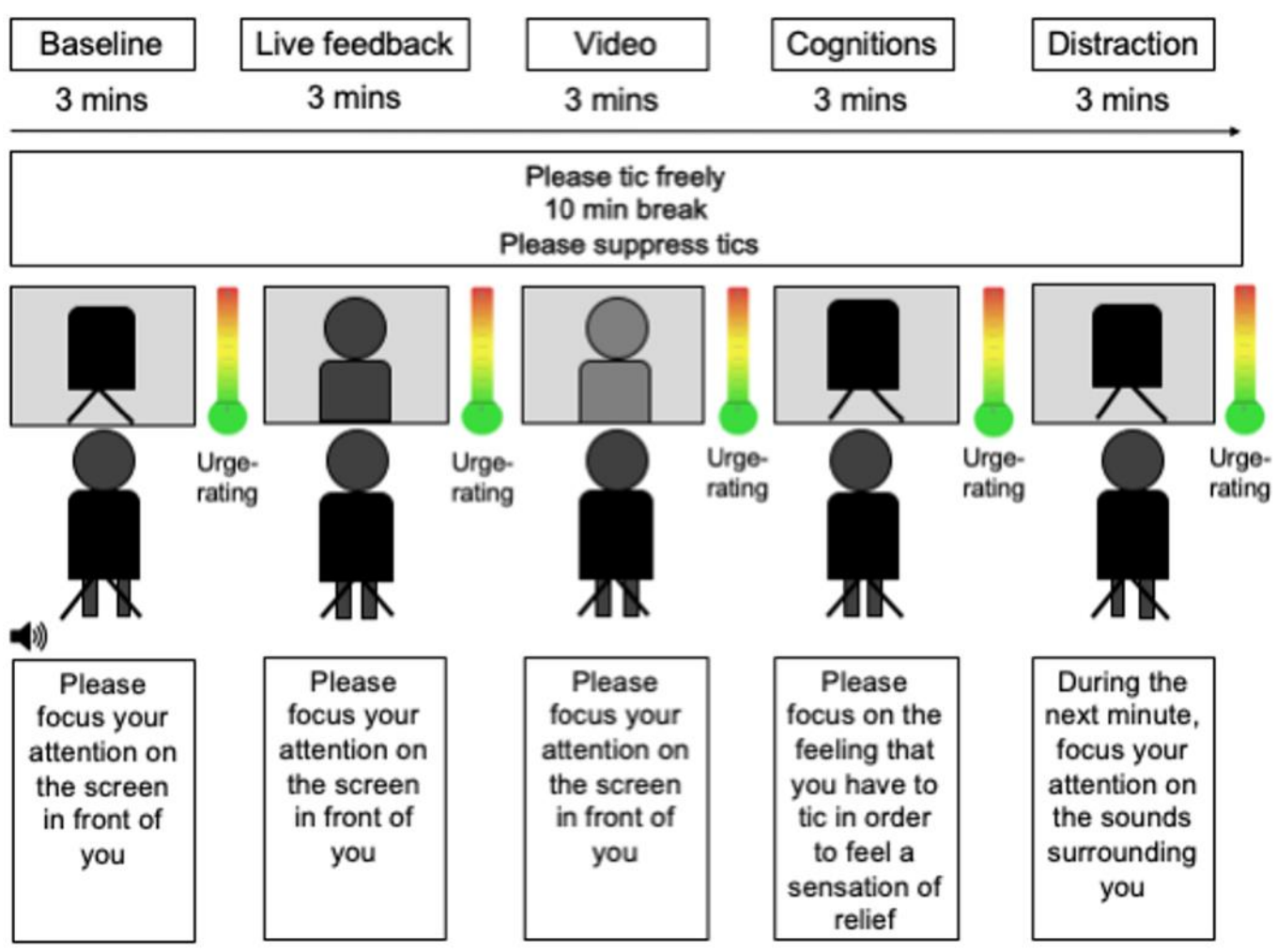

5 Figure 1. Study design

6 Patients sat in front of a computer screen, which displayed a picture of the empty room and

7 the chair the participant was sitting in (baseline, cognitions, distraction), a real-time video

8 feedback showing the patient (live feedback) or a pre-recorded video of the patient, recorded

9 in the live feedback condition (video). During each $3 \mathrm{~min}$ attention condition, patients were

10 instructed six times to pay attention to specific stimuli. Tics were recorded with a video

11 camera, urges were assessed using the urge-thermometer after each attention condition. Pupil

12 width was recorded during each attention condition. 

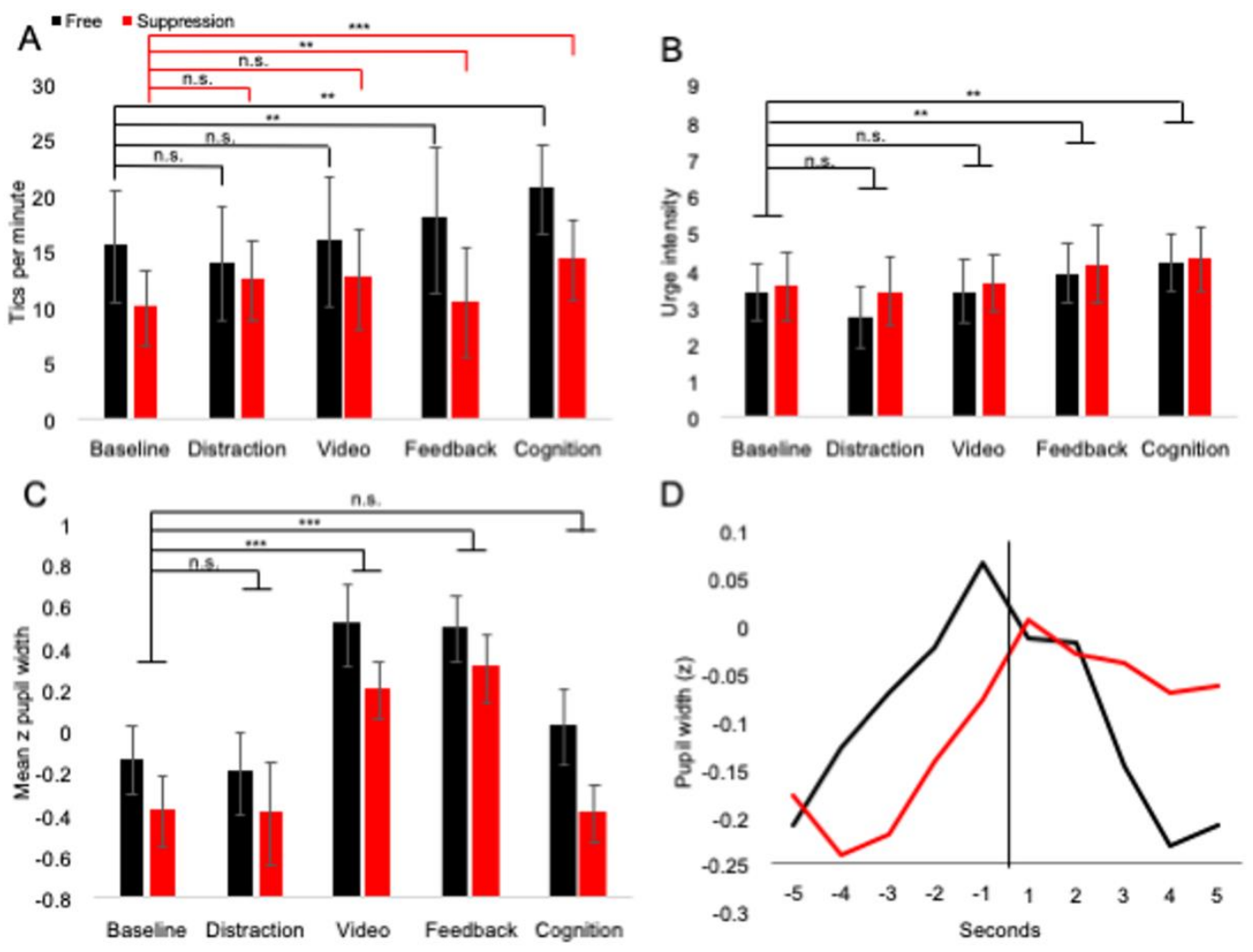

Figure 2.

A: Tics per minute across the five different attention conditions. In the free ticcing condition (grey), patients ticced significantly more often when they watched a live video feedback of themselves and when they thought about their own tics compared to the baseline. In the suppression condition (red), patients ticced significantly more often when they saw a video of their tics or thought about their specific tic-triggering situations.

B: Urge ratings across the five different attention conditions. Patients rated their urges to be higher when they saw a live video feedback of themselves and when they thought about tics. Urge intensity in both the baseline and suppression conditions behaved in the same manner as tic frequency during the baseline condition.

C: Average pupil width across the five attention conditions. Pupils were significantly wider when patients saw a live video feedback of their tics and when they watched a previously recorded video of their tics. Pupils were overall wider during the free block than the suppression block. Pupil data was z-standardized (mean $=0, \mathrm{SD}=1$ ).

D: Average pupil width around single tics during the free and the suppression block from 5 seconds prior to the tic to 5 seconds post ticcing. Logistic regressions showed a significant quadratic distribution (increase, then decrease) in the free block and a significant cubic distribution (increase prior to tic, then levelling off) in the suppression condition. 
Running title: ATTENTION MODULATES TIC-RELATED SYMPTOMS

1 
Running title: ATTENTION MODULATES TIC-RELATED SYMPTOMS

\section{Supplementary material}

2

3 The "Thinking About Tics Inventory" (THAT), German version. 
2 Bitte geben Sie an, inwieweit die folgenden Gedanken Ihrer Erfahrung nach bei Ihnen

3 Tics auslösen

4

\begin{tabular}{|c|c|c|c|}
\hline & Immer & Manchmal & Nie \\
\hline Die Erwartung dass Sie ticcen könnten & 1 & 2 & 3 \\
\hline Im Allgemeinen über Tics nachdenken & 1 & 2 & 3 \\
\hline $\begin{array}{l}\text { Darüber nachdenken, dass Andere Sie beim ticcen } \\
\text { beobachten }\end{array}$ & 1 & 2 & 3 \\
\hline $\begin{array}{l}\text { Der Gedanke, dass Sie ticcen müssen um Erleichterung zu } \\
\text { empfinden }\end{array}$ & 1 & 2 & 3 \\
\hline Über Ihre Tics reden & 1 & 2 & 3 \\
\hline $\begin{array}{l}\text { Wissen, dass Sie mit Menschen zusammen sein werden, } \\
\text { die erwarten dass Sie ticcen }\end{array}$ & 1 & 2 & 3 \\
\hline Sich fragen ob die Tics sie bei Aktivitäten stören werden & 1 & 2 & 3 \\
\hline Sich fragen, ob Sie immer Tics haben werden & 1 & 2 & 3 \\
\hline Sich fragen, ob die Tics in Zukunft schlimmer werden & 1 & 2 & 3 \\
\hline Wissen, dass Sie die Erlaubnis haben zu ticcen & 1 & 2 & 3 \\
\hline Wissen, dass andere Menschen keine Tics haben & 1 & 2 & 3 \\
\hline Denken, dass Sie Tics unterdrücken müssen & 1 & 2 & 3 \\
\hline Wissen, dass Sie nicht ticcen sollten & 1 & 2 & 3 \\
\hline Sich wünschen, keine Tics zu haben & 1 & 2 & 3 \\
\hline Denken, dass Tics Ihr Image kaputt machen & 1 & 2 & 3 \\
\hline $\begin{array}{l}\text { Denken, dass Sie merkwürdig und anders wirken aufgrund } \\
\text { Ihrer Tics }\end{array}$ & 1 & 2 & 3 \\
\hline $\begin{array}{l}\text { Darüber nachdenken, wie anstrengend es für Sie ist zu } \\
\text { ticcen }\end{array}$ & 1 & 2 & 3 \\
\hline Die Tics als nervig oder qualvoll zu empfinden & 1 & 2 & 3 \\
\hline Wenn Sie sich selber ticcen sehen & 1 & 2 & 3 \\
\hline Jemand anders ticcen sehen & 1 & 2 & 3 \\
\hline Denken, dass Ihre Tics Sie schlecht dastehen lassen & 1 & 2 & 3 \\
\hline Bemerken, dass Sie eine Weile nicht geticct haben & 1 & 2 & 3 \\
\hline
\end{tabular}


Running title: ATTENTION MODULATES TIC-RELATED SYMPTOMS

Supplementary table 1. Descriptive data German and English THAT items

\begin{tabular}{|c|c|c|}
\hline THAT items & $\begin{array}{l}M \pm S D \\
\text { current } \\
(n=23)\end{array}$ & $\begin{array}{l}\mathrm{M} \pm \mathrm{SD} \\
\text { O'Connor et al., } \\
(\mathbf{2 0 1 4})\end{array}$ \\
\hline Anticipating that you might tic & $1.87(0.63)$ & $1.85(0.68)$ \\
\hline Thinking in general about your tics & $1.85(0.55)$ & $1.85(0.63)$ \\
\hline Thinking others will observe you ticcing & $1.89(0.80)$ & $1.95(0.74)$ \\
\hline The idea that you must tic to feel relief & $1.61(0.72)$ & $1.82(0.65)$ \\
\hline Talking about your tics & $2.09(0.67)$ & $2.03(0.71)$ \\
\hline Knowing you will be with people who expect you to tic & $2.26(0.62)$ & $2.30(0.67)$ \\
\hline Wondering if your tics will interfere with your activities & $2.17(0.65)$ & $2.15(0.70)$ \\
\hline Asking yourself if you will always have tics & $2.09(0.79)$ & $2.15(0.70)$ \\
\hline Asking yourself whether your tics will get worse & $2.00(0.74)$ & $2.25(0.65)$ \\
\hline Knowing you have permission to tic & $2.22(0.67)$ & $2.17(0.76)$ \\
\hline Knowing that other people do not have tics like you & $2.48(0.59)$ & $2.43(0.72)$ \\
\hline Thinking you will need to suppress a tic & $1.87(0.69)$ & $1.80(0.73)$ \\
\hline Knowing you should not be ticcing & $1.87(0.69)$ & $1.85(0.73)$ \\
\hline Wishing you did not have tics & $2.04(0.88)$ & $2.05(0.74)$ \\
\hline Thinking tics spoil your image & $2.61(0.58)$ & $2.07(0.75)$ \\
\hline Thinking you appear odd and different due to your tics & $2.13(0.69)$ & $2.07(0.75)$ \\
\hline Dwelling on how your tics tire you out & $2.00(0.90)$ & $2.10(0.70)$ \\
\hline Finding your tics annoying or distressing & $2.04(0.82)$ & $1.83(0.69)$ \\
\hline Seeing yourself tic & $1.96(0.71)$ & $1.82(0.70)$ \\
\hline Observing someone else tic & $2.17(0.72)$ & $2.30(0.78)$ \\
\hline Thinking your tics make you look bad & $2.32(0.72)$ & $2.13(0.74)$ \\
\hline Noticing you have not ticced for some time & $1.87(0.63)$ & $1.95(0.64)$ \\
\hline
\end{tabular}

The table shows means and standard deviations $(\mathrm{M} \pm \mathrm{SD})$ for each of the Thinking About

4 Tics Inventory (THAT) items (left column) for the German version (middle column) and the

5 English version (right column), published by O'Connor and colleagues, in 2014. Participants

6 are instructed to indicate how often these thoughts triggered tics. Note that all items presented

7 here used the original coding, that is, lower values reflect more agreement (always $=1$,

8 sometimes $=2$, never $=3$ ). Items that the tic-related thoughts in this experiment were based

9 on are indicated in italics. 